This item was submitted to Loughborough's Research Repository by the author.

Items in Figshare are protected by copyright, with all rights reserved, unless otherwise indicated.

\title{
Electron trapping in radio-frequency atmospheric-pressure glow discharges
}

PLEASE CITE THE PUBLISHED VERSION

PUBLISHER

(C) American Institute of Physics

VERSION

VoR (Version of Record)

LICENCE

CC BY-NC-ND 4.0

\section{REPOSITORY RECORD}

Liu, D.W., J.J. Shi, and Michael G. Kong. 2019. "Electron Trapping in Radio-frequency Atmospheric-pressure Glow Discharges”. figshare. https://hdl.handle.net/2134/5185. 
This item was submitted to Loughborough's Institutional Repository (https://dspace.lboro.ac.uk/) by the author and is made available under the following Creative Commons Licence conditions.

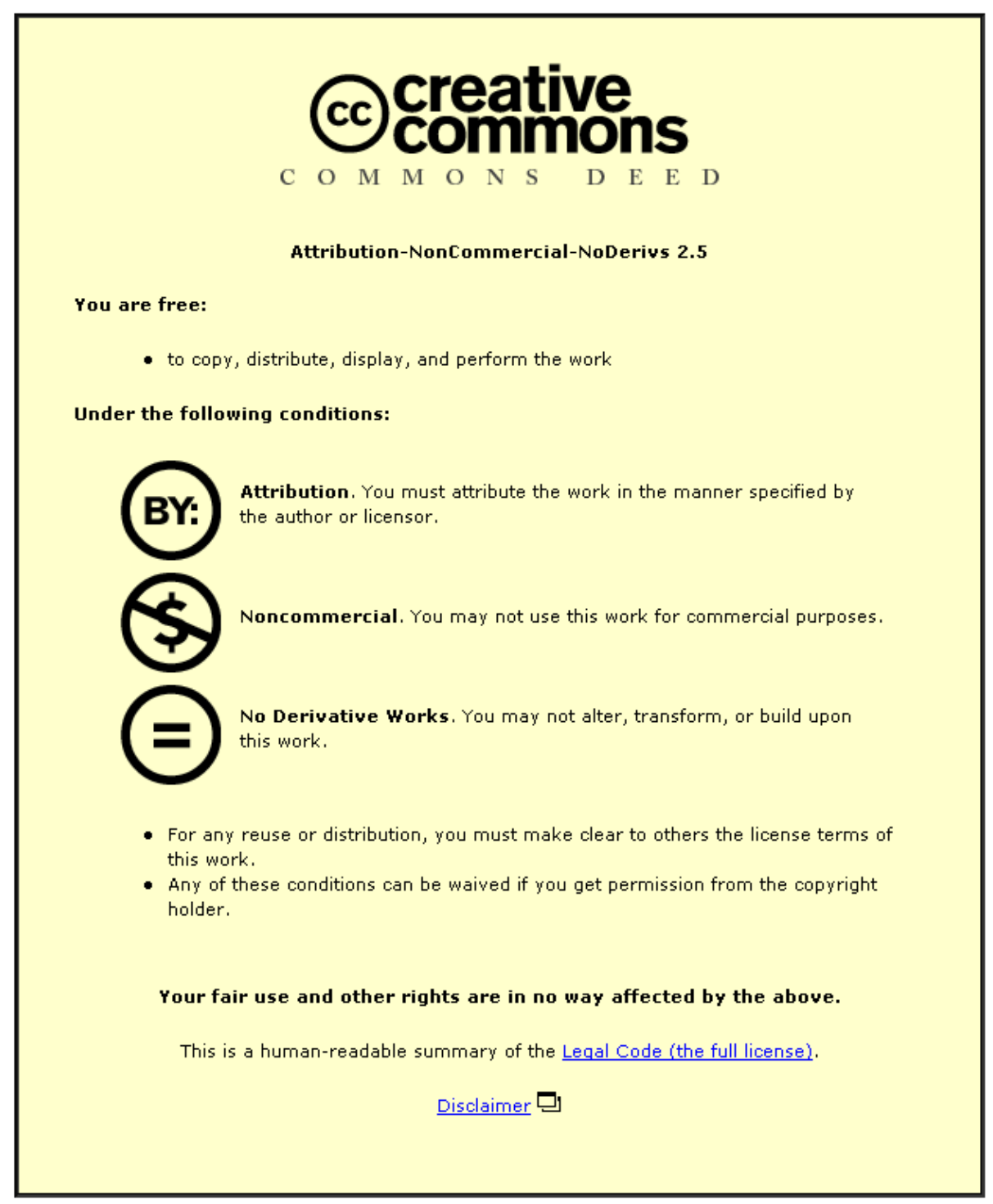

For the full text of this licence, please go to: http://creativecommons.org/licenses/by-nc-nd/2.5/ 


\title{
Electron trapping in radio-frequency atmospheric-pressure glow discharges
}

\author{
D. W. Liu, J. J. Shi, and M. G. Kong ${ }^{\text {a) }}$ \\ Department of Electronic and Electrical Engineering, Loughborough University, Loughborough, \\ Leicestershire LE11 3TU, United Kingdom
}

(Received 4 September 2006; accepted 27 November 2006; published online 25 January 2007)

\begin{abstract}
In this letter, the authors present experimental evidence of electron trapping in radio-frequency (rf) atmospheric-pressure glow discharges. By linking electron density to nanosecond plasma images and optical emission spectroscopy, they show that electron trapping occurs under most discharge conditions. The level of electron trapping increases with increasing discharge current or/and increasing excitation frequency, and manifests itself in the change of the differential conductivity at the point of the gas breakdown. Finally, they demonstrate that electron trapping is largely related to whether the half rf period is shorter than the electron transition time across the electrode gap.

(C) 2007 American Institute of Physics. [DOI: 10.1063/1.2425045]
\end{abstract}

Large-volume glow discharges generated at atmospheric pressure represent one of the most important topics in plasma physics, since they offer a unique chamberless route to material processing. ${ }^{1}$ Commonly known as atmosphericpressure glow discharges (APGDs), a vast majority of their studies over the past 20 years has been focused on their applications including nanoscience, ${ }^{2}$ medicine, ${ }^{3,4}$ space exploration, ${ }^{5}$ and display technologies. ${ }^{6}$ Lagging much behind are fundamental studies that are yet to enable a coherent understanding of APGD physics and chemistry. At radio frequencies (rfs) of $1-60 \mathrm{MHz}$, this has been partly addressed through a series of recent experimental ${ }^{7-9}$ and computational studies. ${ }^{10-12}$ For example, electron trapping has been proposed theoretically as a primary mechanism for generating $\mathrm{rf}$ APGD. ${ }^{10,11}$ Compared to the half rf period, the electron transition time across the electrode gap is considered to be long and hence most electrons could be trapped in the electrode gap by the fast oscillating rf voltage. This would allow the balance between electron creation and loss to be achieved at a comparatively low rf voltage, thus suppressing the glow-to-arc transition. While electron trapping is plausible as a possible mechanism for generating rf APGD, it remains a theoretical prediction and its validity has not so far been supported with experimental evidence. In this letter, we present an experimental study of electron trapping phenomena in rf APGD.

Our rf APGD system employed two parallel stainlesssteel electrodes, one being a round disk of $2 \mathrm{~cm}$ in diameter and the other being a rectangular plate of $4 \times 8 \mathrm{~cm}^{2}$. Hence, the electrode surface area was $3.1 \mathrm{~cm}^{2}$. The two electrodes were naked without any dielectric barriers, and their separation distance was fixed at $0.24 \mathrm{~cm}$. The electrode unit was housed within a Perspex box fed with a through helium flow at 5 slm (standard liters per minute). The Perspex box was not air tight, and so oxygen and nitrogen were present. A rf power amplifier (Amplifier Research 150A100B) and a function generator were used to deliver a rf voltage at $1-10 \mathrm{MHz}$ to the electrode unit via a homemade matching network. Current and voltage were measured by a wideband current probe

\footnotetext{
${ }^{\text {a) }}$ Author to whom correspondence should be addressed; electronic mail:
} m.g.kong@lboro.ac.uk
(Tektronix P6021), a wideband voltage probe (Tektronix P6015A), and a digital oscilloscope (Tektronix TDS 5054B). Plasma images were taken with an intensified chargecoupled device (iCCD) camera (Andor i-Star DH720) and a pulse generator for triggering the iCCD. Optical emission spectrum was measured using a spectrometer system (Andor Shamrock) with a focal length of $0.3 \mathrm{~m}$ and a grating of 600 grooves/mm.

For all our experiments, the discharge current was sinusoidal with one positive peak and one negative peak in each rf cycle of the applied voltage. ${ }^{7}$ Figure 1 shows the currentvoltage characteristics at five different frequencies from prebreakdown (indicated with dotted lines), through glow discharge (indicated with markers), to the point immediately before plasma constriction or arcing (as the last point on each curve). The prebreakdown sections of all curves go through the origin in straight lines. At $10 \mathrm{MHz}$, the transition from the prebreakdown regime to the discharge regime is marked with a step change in the slope of the voltage-current curve, signifying a step change in the differential conductivity of the discharge plasma. This is similar to reported observations of most rf APGD generated typically at $13.56 \mathrm{MHz} .^{7-9,13}$ For future reference, we refer this transition point to as the breakdown point. In the cases of 5 and $7 \mathrm{MHz}$, a similar but smaller step change is evident at the breakdown point. As the excitation frequency reduces below $4 \mathrm{MHz}$, the step change becomes negligible as shown in Fig. 1. This invariance from

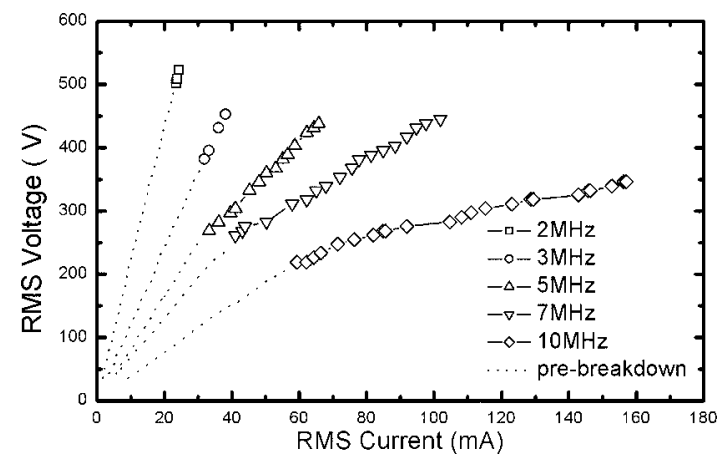

FIG. 1. Current-voltage characteristics from prebreakdown (dotted lines), through glow modes (markers), to plasma constriction (immediately after the last point of each curve). 
the prebreakdown to the discharge regimes has not been reported before and is characteristic in APGD at low radio frequencies $(<4 \mathrm{MHz})$. Therefore, the current-voltage characteristics in rf APGD manifest themselves differently at different frequencies.

To interpret the observations in Fig. 1, we model the rf APGD using an equivalent circuit consisting of a capacitor and a series resistor. ${ }^{14}$ The capacitor of capacitance $C_{s}$ models the electron-depleted sheath region, whereas the resistor of resistance $R_{p}$ models the quasineutral region of the bulk plasma. Therefore, the equivalent impedance of the gas gap is $Z_{g}=R_{p}-j / \omega C_{s}$ with $\omega=2 \pi f$ being the angular excitation frequency. In the prebreakdown regime, the impedance becomes $Z_{g}=j / \omega C_{g}$ with $C_{g}$ being the capacitance of the unionized gas gap. If most electrons generated in the discharge regime can easily reach the electrodes during one half rf period, $\tau_{\mathrm{rf}}$, their presence in the gas gap is short lived and is unlikely to increase significantly the average electrical conductivity of the gas gap. As a result, $Z_{g} \approx-j / \omega C_{g}$ and the electrical characters of the gas gap remain similar to those in the prebreakdown regime. Assuming an electric field of $500 \mathrm{~V} / 0.24 \mathrm{~cm}$ at the breakdown point for the $2 \mathrm{MHz}$ case and an electron mobility of $1132 \mathrm{~cm}^{2} \mathrm{~V}^{-1} \mathrm{~s}^{-1}$, the electron transition time across the electrode gap of $0.24 \mathrm{~cm}$ is found to be $\tau_{e}=102 \mathrm{~ns}$. This is significantly below $\tau_{\mathrm{rf}}=250 \mathrm{~ns}$ at $2 \mathrm{MHz}$, suggesting that most generated electrons can easily reach the electrodes during one half of the rf cycle. Electron trapping is therefore temporary and the gas impedance is approximately $Z_{g} \approx-j / \omega C_{g}$. This may be responsible for the invariance in the differential conductivity in the $2 \mathrm{MHz}$ case in Fig. 1. As the frequency increases to $3 \mathrm{MHz}$, our calculation shows that $\tau_{\mathrm{rf}}=166 \mathrm{~ns}$ and $\tau_{e}=134 \mathrm{~ns}$ at the breakdown point, much closer to each other than those at $2 \mathrm{MHz}$. As $\tau_{e}<\tau_{\mathrm{rf}}$, many generated electrons can still reach the electrodes and the gas gap can retain its electrical conductivity in the prebreakdown regime. Again, this explains why the slope of the voltage-current curve changes little at $3 \mathrm{MHz}$. Raising the frequency to $5 \mathrm{MHz}$, we find that $\tau_{e}=195 \mathrm{~ns}$ is now larger than $\tau_{\mathrm{rf}}=100 \mathrm{~ns}$. This suggests that many electrons can no longer reach the electrodes during one half-period and will be trapped in the electrode gap. Further frequency increment leads to $\tau_{e}$ becoming increasingly larger than $\tau_{\mathrm{rf}}$ and so a greater level of electron trapping. If sufficient electrons are trapped, they will increase the electrical conductivity of the gas gap and change the slope of the voltage-current curves as in the cases of 5, 7, and $10 \mathrm{MHz}$. In other words, a step change in the slope of the voltage-current curve is indicative of electron trapping. Figure 1 suggests a progressively increasing level of electron trapping with increasing frequency. The voltage-current phase shift at the breakdown point was found experimentally to decrease almost linearly from $89^{\circ}$ to $77^{\circ}$ as the frequency was increased from 2 to $10 \mathrm{MHz}$. This is a result of more electrons being trapped in the gas gap thus making the gas gap more conductive.

To further support the above argument of electron trapping, it is most logical to measure the electron density. For rf APGD in helium, the electron density is below $10^{12} \mathrm{~cm}^{-3}$ typically ${ }^{10-12}$ for which the Stark broadening technique is inadequate. Langmuir probes are also inappropriate for atmospheric plasmas. These highlight the current lack of suitable techniques for measuring the electron density of APGD. As an alternative, we studied possible optical signatures of electrons. In Fig. 2(a), five plasma images with an exposure Downloaded 20 Aug 2009 to 158.125.80.71. Redistribution subject
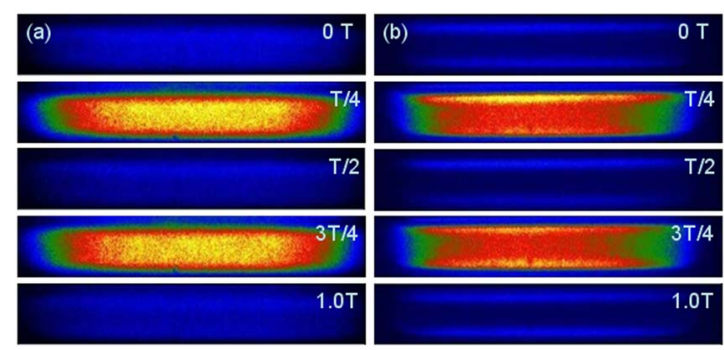

FIG. 2. (Color online) Nanosecond images of a $10 \mathrm{MHz}$ rf APGD over one complete rf cycle and at (a) $70.0 \mathrm{~mA}$ and (b) $140.5 \mathrm{~mA}$.

time of $10 \mathrm{~ns}$ are shown for the $10 \mathrm{MHz}$ case at $I_{\mathrm{rms}}=70.0 \mathrm{~mA}$. They are periodic at the driving frequency with the brighter images at $T / 4$ and $3 T / 4$ taken at the discharge current peaks. Their spatial profile is bell-like, spreading over the electrode gap and indicating a volumetric discharge. This suggests that the excited plasma species are produced volumetrically and are most numerous in the gap center. When the discharge current is doubled to $I_{\mathrm{rms}}$ $=140.5 \mathrm{~mA}$, the discharge dynamics remain periodic at $10 \mathrm{MHz}$ but the plasma structure changes to a doublehumped profile as shown in Fig. 2(b). The optical emission is no longer volumetric but localized near the electrodes. At $T / 4$, a bright thin layer is evident near the top electrode and it reappears near the bottom electrode at $3 T / 4$. This bright thin layer is likely to be the negative glow, formed by a localized concentration of excited plasma species which in turn are generated by energetic electrons. These spatial profiles are more clearly shown in Fig. 3 as a function of the interelectrode position. As the current increases, the spatial profile of optical emission evolves from a bell-like shape to a double-humped shape. As electrons are largely responsible for generating excited plasma species, Fig. 3 suggests that energetic electrons are also trapped and their trapping enhances with increasing current. Since optical emission remains visible in all images in Fig. 2, electron trapping is permanent. Also as $I_{\text {rms }}$ increased from 70.0 to $140.5 \mathrm{~mA}$, the voltage-current phase shift in Figs. 2 and 3 was found to decrease from $77^{\circ}$ to $73^{\circ}$ indicating a more conductive gas gap and hence more trapped electrons at the large current. Therefore with a large discharge current, the level of electron trapping is likely to be greater and the trapped electrons are likely to be more energetic. In turn, the energetic trapped electrons enhance gas ionization and lead to much brighter

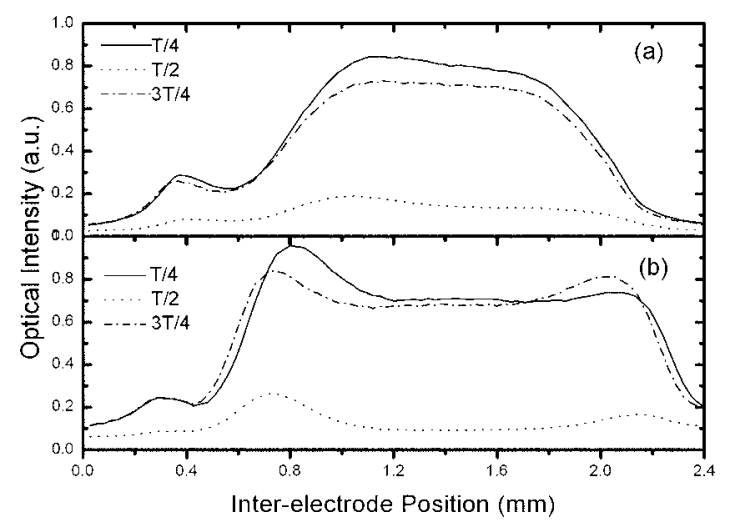

FIG. 3. Spatial profile of the total optical emission at $10 \mathrm{mHz}$ across the electrode gap at (a) $70.0 \mathrm{~mA}$ and (b) $140.5 \mathrm{~mA}$. The power electrode was

located at $x=0.0 \mathrm{~mm}$.

located at $x=0.0 \mathrm{~mm}$.
AIP license or copyright; see http://apl.aip.org/apl/copyright.jsp 


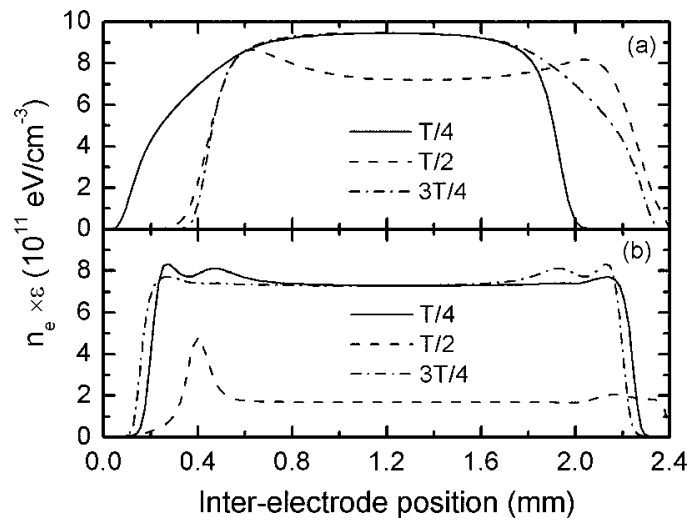

FIG. 4. Spatial temporal profiles of the product of the electron density and the mean electron energy at (a) $10 \mathrm{~mA} / \mathrm{cm}^{2}$ and (b) $50 \mathrm{~mA} / \mathrm{cm}^{2}$.

optical emission at larger currents. It is also worth noting the small hump near $x=0 \mathrm{~mm}$, which is a dc bias.

The link of nanosecond plasma images to energetic electrons can be further supported by optical emission spectroscopy. For all experiments reported here, the most intense emission is from the helium line at $706 \mathrm{~nm}$ and the nitrogen line at $391 \mathrm{~nm}$ (data not shown). The helium line at $706 \mathrm{~nm}$ indicates the presence of either energetic electrons or $\mathrm{He}_{2}^{+}$ ions and low energy electrons. ${ }^{15}$ The $391 \mathrm{~nm}$ emission line is known to be resulted from $\mathrm{N}_{2}^{+}\left(B^{2} \Sigma_{u}^{+}, v_{b}=0\right)$ transition to $\mathrm{N}_{2}^{+}$ $\left(X^{2} \Sigma_{u}^{+}, v_{x}=0\right)$, and contains signatures of helium metastables and $\mathrm{He}_{2}^{+}$ions. ${ }^{15}$ As the most numerous ionic species are $\mathrm{He}_{2}^{+}$ ions in helium rf APGD, ${ }^{11,16}$ the combination of the $706 \mathrm{~nm}$ line and the $391 \mathrm{~nm}$ line is an excellent indicator of the energetic electrons and the most significant ions. Therefore, the spatial profile of optical emission in Fig. 3 is likely to mirror that of energetic electrons. Consequently, the bell-like profile at $I_{\mathrm{rms}}=70.0 \mathrm{~mA}$ suggests volumetric trapping of energetic electrons across the electrode gap whereas the doublehumped profile at $I_{\mathrm{rms}}=140.5 \mathrm{~mA}$ suggests localized trapping of energetic electrons near the electrodes.

To relate to the original theoretical prediction, we performed one-dimensional simulation of the $10 \mathrm{MHz}$ APGD using a fluid model. ${ }^{11,12}$ Figure 4 shows the spatial-temporal profile of the product of the electron density and the mean electron energy as a measure of energetic electrons. In a broad term, these profiles match well those in Fig. 3. The density-energy product at the large current density is larger than that at the small current density, consistent with that the optical emission was much more intense at $140.5 \mathrm{~mA}$ than at $70.0 \mathrm{~mA}$ (data not shown). This comparison confirms that electron trapping does occur in rf APGD and their level increases progressively with increasing discharge current.

At lower excitation frequencies, electron trapping was found to be weaker. Figure 5 shows a sequence of five plasma images of the $3 \mathrm{MHz}$ APGD of Fig. 1 again with an exposure time of $10 \mathrm{~ns}$. They were taken at $I_{\mathrm{rms}}=36.1 \mathrm{~mA}$, corresponding to the point immediately before arcing in Fig.

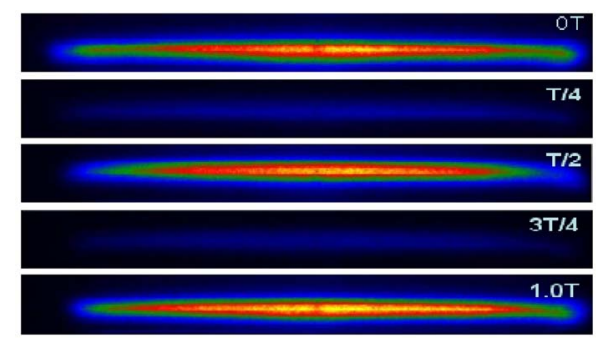

FIG. 5. (Color online) Nanosecond images of a $3 \mathrm{MHz}$ rf APGD over one complete rf cycle and at $36.1 \mathrm{~mA}$.

1 and representing the maximum level of electron trapping at $3 \mathrm{MHz}$. It is clear that electron trapping has a bell-like profile similar to that in Fig. 2(a), suggesting a low level of electron trapping even though the discharge current is the largest before arcing. Its bell-like profile is sustained over a narrow central region of the electrode gap and as such is less significant than that in the $10 \mathrm{MHz}$ case.

In summary, we have presented experimental evidence of electron trapping in rf APGD. By linking electron density to nanosecond plasma images and optical emission at 391 and $706 \mathrm{~nm}$, electron trapping has been shown to persist at 1-10 MHz. It has also been demonstrated that electron trapping enhances with increasing discharge current or/and increasing excitation frequency. In terms of its frequency dependence, the level of electron trapping has been found to manifest itself in the change of the differential conductivity at the breakdown point. Finally, the main reason for electron trapping has been shown to be related to whether $\tau_{e}>\tau_{\mathrm{rr}}$.

This work was funded in part by the Engineering and Physical Science Research Council (U.K.).

${ }^{1}$ J. R. Roth, Industrial Plasma Engineering Principles (Institute of Physics, Philadelphia, 1995), Vol. 1, pp. 453-460.

${ }^{2}$ H. Shirai, T. Kobayashi, and Y. Hasegawa, Appl. Phys. Lett. 87, 143112 (2005).

${ }^{3}$ M. Vleugels, G. Shama, X. T. Deng, E. Greenacre, T. Brocklehurst, and M. G. Kong, IEEE Trans. Plasma Sci. 33, 824 (2005).

${ }^{4}$ W. S. Lai, H. R. Lai, S. P. Kuo, O. Tarasenko, and K. Levon, Phys. Plasmas 12, 023501 (2005).

${ }^{5}$ Y. Takao and K. Ono, Plasma Sources Sci. Technol. 15, 211 (2006).

${ }^{6}$ J. G. Eden, Proc. IEEE 94, 567 (2006).

${ }^{7}$ J. J. Shi, X. T. Deng, R. Hall, J. D. Punnett, and M. G. Kong, J. Appl. Phys. 94, 6303 (2003).

${ }^{8}$ A. P. Yalin, Z. Q. Yu, O. Stan, K. Hoshimiya, A. Rahman, V. K. Surla, and G. J. Collins, Appl. Phys. Lett. 83, 2766 (2003).

${ }^{9}$ K. Niemi, V. Schulz-von der Gathen, and H. F. Dobele, Plasma Sources Sci. Technol. 14, 375 (2005).

${ }^{10}$ X. Yuan and L. L. Raja, IEEE Trans. Plasma Sci. 31, 495 (2003).

${ }^{11}$ J. J. Shi and M. G. Kong, J. Appl. Phys. 97, 023306 (2005).

${ }^{12}$ J. J. Shi and M. G. Kong, Phys. Rev. Lett. 96, 105009 (2006).

${ }^{13}$ J. Park, I. Henins, H. W. Herrmann, G. S. Selwyn, and R. F. Hicks, J. Appl. Phys. 89, 20 (2001).

${ }^{14}$ J. J. Shi and M. G. Kong, J. Appl. Phys. 94, 5504 (2003).

${ }^{15}$ G. Nersisyan and W. G. Graham, Plasma Sources Sci. Technol. 13, 582 (2004).

${ }^{16}$ J. J. Shi and M. G. Kong, IEEE Trans. Plasma Sci. 33, 624 (2005). 